\section{Optimal design of honeycombs}

SIR - In 1964, L. F. Toth delivered an entertaining lecture entitled "What the bees know and what they do not know"1. In it, he analysed the geometry of the bees' honeycomb in terms of the efficient use of wax and hence the minimization of the total area of the surfaces involved. When viewed in such terms, the architecture used by the bees is not quite
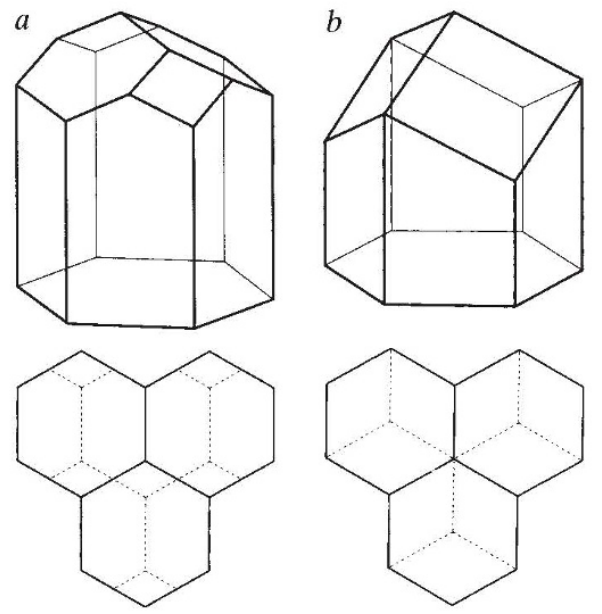

FIG. 1 Alternative structures for the honeycomb interface. $a$, Toth structure; $b$, beehive structure. optimal, but the possible improvement on the bees' design is very small.

Toth went on to describe a superior design for the honeycomb interface, while noting that there was no proof that his choice was in any sense optimal in the biological context. It is closely related to Kelvin's proposed optimal division of space into periodic cells ${ }^{2}$

We have undertaken an experiment which demonstrates Toth's proposed structure, invites a generalization of his problem to foam structures of finite liquid content, and shows that the bees' choice is reinstated as superior whenever the liquid fraction is sufficiently high. The structures in question are illustrated in Fig. 1.

We can explore the analogous problem for liquid foam by introducing equal-sized bubbles (here with approximately $2-\mathrm{mm}$ diameter) of a detergent solution between two glass plates, the plate separation being such that a double layer of bubbles is trapped. Each layer forms an array of hexagonal cells. A foam of low liquid content consists of thin films whose junctions are readily idealized as lines. In this case, which corresponds to Toth's geometrical picture, we observe the structure proposed by him (Fig. 2).

If we wet the foam by the addition of more liquid, the junctions between films are thickened to form what are called Plateau borders. The conditions under which surface energy is to be minimized are accordingly changed. What happens

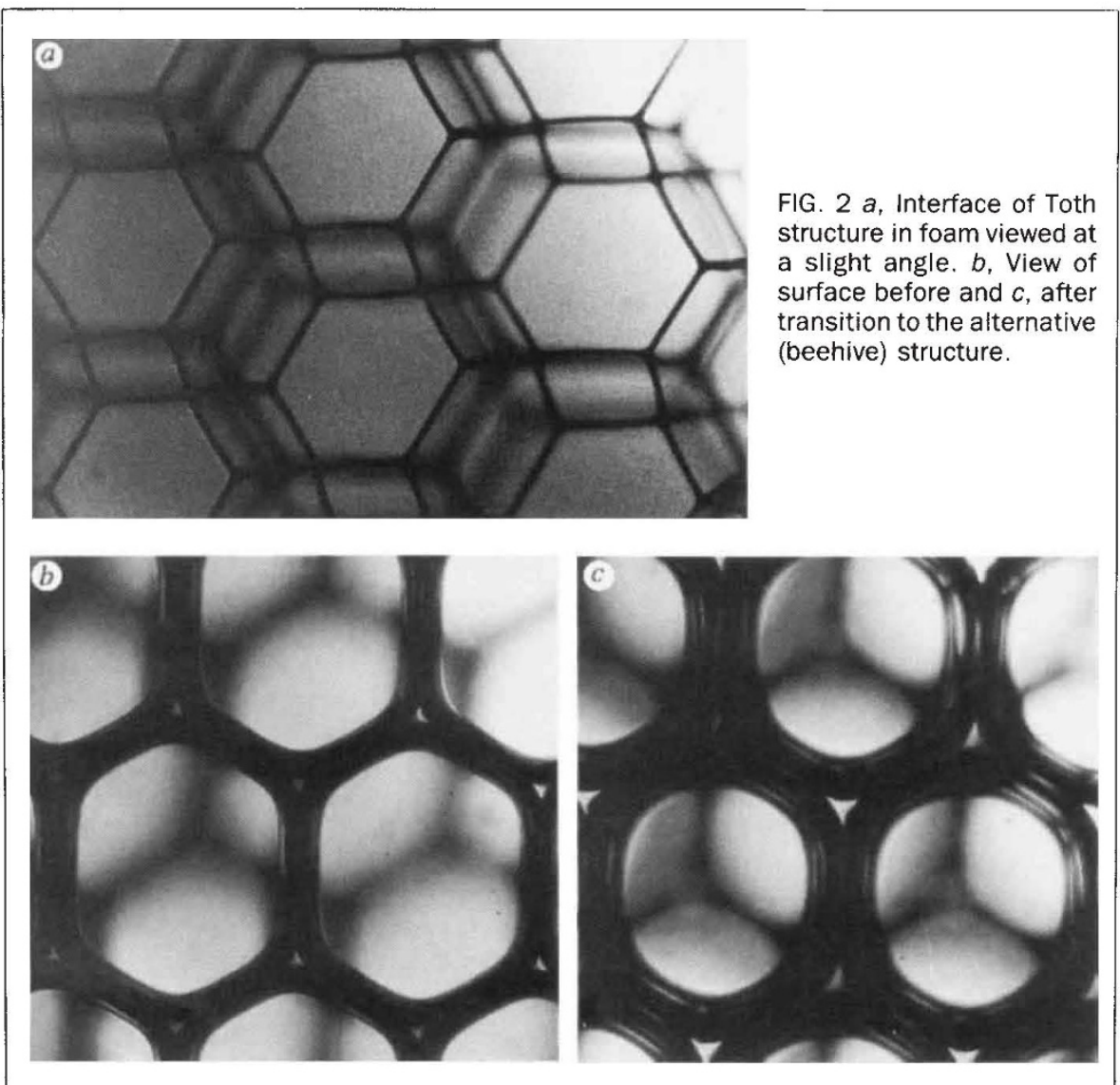

as the foam is progressively wetted is quite dramatic. At a certain point the Toth structure becomes unstable and there is a sudden switch to the configuration favoured by the bees (Fig. 2). Such a switch also takes place in the reverse direction, as liquid is removed.

This structural transition relates to current ideas of foam structure in three dimensions ${ }^{3,4}$. It will provide a particularly convenient test of quantitative theories of topological changes due to instabilities, and will be further analysed by us in that context.

D. Weaire

R. Phelan

Department of Pure and Applied

Physics,

Trinity College, Dublin, Ireland

1. Toth, L. F. Bull. Am. math. Soc. 70, 469-481 (1964).

2. Thomson, W. (Lord Kelvin) Phil. Mag. 24, 503-514 (1887)

3. Weaire, D. Phil. Mag. Lett. (in the press).

4. Weaire, D. \& Phelan, R. Phil. Mag. Lett. (in the press)

\section{How populations persist}

SIR - How much suitable habitat or resources does a population require to persist or, to put it another way, what is the eradication threshold of the population? According to one approach first developed in epidemiology ${ }^{1}$, the simplest estimate of the eradication threshold of a disease (specifically, how large a fraction of the population, failing to be covered by a vaccination programme, would allow the disease to persist) is the observed fraction susceptible to the disease at equilibrium. In more general terms, an estimate of the eradication threshold for a population is simply the unused amount of its limiting resource (susceptible individuals being the limiting resource for the multiplication of a disease organism).

Lande $^{2-4}$ derived an estimate of the eradication threshold for territorial species, that is, an estimate of the proportion of a region which must consist of suitable territories if the population is to persist, and applied this estimate to the northern spotted owl, Strix occidentalis caurina, which has been the subject of intense interest by conservationalists. Lande's estimate of the threshold is the fraction of sites which are currently unoccupied although they are suitable (see ref. 5 for a more detailed discussion). Given that suitable breeding territories were taken to be the limiting resource for territorial species, this is the epidemiological estimate. The fact that the many biological differences between owls and say, weasles, do not seem to affect the eradication threshold estimate prompts the investigation of a rather different ecological model to probe its generality further. 